\title{
APROXIMACIÓN AL PENSAMIENTO EDUCATIVO DE RODOLFO KUSCH
}

\author{
Mario Mejía Huamán \\ Universidad Ricardo Palma \\ mejiahuaman@gmail.com
}

\begin{abstract}
RESUMEN
En la ponencia tratamos de inferir una filosofía de la educación para América Andina a partir del pensamiento de Rodolfo Kusch, sobre todo orientado a la educación del indígena y su descendencia mestiza, que somos nosotros, a partir de lo primigenio de la concepción propia del mundo que subyace en el fondo de nuestra existencia, pero, que ha sido ocultada por la inmersión a la cultura occidental que hemos sufrido desde la conquista de América hasta el momento. La consecuencia lógica es habernos acostumbrado a mirar nuestra realidad y pensar sobre ella, con anteojos ajenos, buscar solucionar nuestros problemas sin tomar en cuenta para nada, el conocimiento propio de nuestra "pacha".
\end{abstract}

\section{PALABRAS CLAVE}

Educación, indígena, Kusch, pacha, cosmovisión.

\begin{abstract}
In the paper we try to infer a philosophy of education for Andean America from the thought of Rodolfo Kusch, especially oriented to the education of the indigenous and their mestizo offspring, which we are, based on the original conception of the world that lies at the bottom of our existence, but that has been hidden by the immersion in Western culture that we have suffered since the conquest of America so far. The logical consequence is to have become accustomed to look at our reality and to think about it, with other people's glasses, to seek to solve our problems without taking into account at all, the own knowledge of our "pacha".
\end{abstract}

\section{KEYWORDS}

Education, indigenous, Kusch, "pacha”, worldview. 


\section{ANTECEDENTES}

El problema de la educación en América Latina ha sido tratado por muchos filósofos, sociólogos, educadores, psicólogos y políticos, entre los que podemos citar como referencia a José Carlos Mariátegui, a Antonio Encinas, Pedro Zulén; A nivel de Latinoamérica, cada país ha tenido y tiene sus filósofos dedicados a la reflexión educativa. Entre muchos otros podemos citar Paulo Freire y a Rodolfo Kusch del hoy que nos ocuparemos.

En los 7 ensayos de Interpretación de la Realidad Peruana, José Carlos Mariátegui hizo una apreciación marxista heterodoxa del problema de la educación y el problema del indio; heterodoxa sostenemos porque El Maestro no aplicó al pie de la letra el marxismo sino como una concepción del mundo. En tal sentido, si bien no rechaza, por ejemplo, la concepción religiosa del indígena, sostiene como marxista que el problema del indio es problema sobre todo económico, de modo que devolviéndole la propiedad de la tierra podríamos lograr que este se inserte en la sociedad oficial.

Mariátegui escribe:

En el proceso de la instrucción pública, como en otros aspectos de nuestra vida, se constata la superposición de elementos extranjeros combinados, insuficientemente aclimatados. El problema está en las raíces mismas de este Perú hijo de la conquista... somos un pueblo en el que conviven, sin fusionarse aún, sin entenderse todavía, indígenas, y conquistadores... el interés de las cuatro quintas partes de la población no juegan casi ningún rol en la formación de la nacionalidad y de sus instituciones. (Mariátegui, 1980, p. 105-106)

El problema del analfabetismo del indio resulta ser, en fin, un problema mucho mayor, que desborda del restringido marco de un plan meramente pedagógico. Cada día se comprueba más que alfabetizar no es educar. La escuela elemental no redime moral y socialmente al indio. El primer paso real hacia su redención, tiene que ser el de abolir la servidumbre. (Ibídem, p. 160)

A pie de página Mariátegui escribe: "El Ministro de Instrucción... en una discurso pronunciado en el Congreso en la legislatura de 1927, ha reconocido la vinculación del problema de la educación indígena y el pro- 
blema de la tierra aceptando una realidad eludida invariablemente por su predecesores en ese cargo".

Antonio Encinas, profesor normalista nacido en Puno que tuvo una amplia formación como profesor y jurisconsulto en el Perú y extranjero defendió que a la escuela se le debía dar una nueva orientación, propuso que ésta fuera la Escuela Social; coincidía con Mariátegui al concebir que la solución al problema indígena no era cuestión pedagógica, de enseñar a leer, a escribir, a contar y rezar, además de recibir información en un idioma que no entiende, sino que la reivindicación del indígena debería consistir en hacerlo un ser productivo en el campo de la economía, lo cual le conduciría a ser partícipe y actor del desarrollo nacional, como lo fue ancestralmente.

La escuela social va a exigir un nuevo maestro, siendo necesaria la revisión de los planes y programas de estudios de la Escuela Normal de Lima, debiéndose enseñar materias que preparen al maestro en la psicología indígena, historia de la evolución política y económica del país, en la cual el sistema de dominación y explotación del indio sea tema de análisis y reflexión, así como la historia desde la visión de los vencidos, planteamiento que sería retomado años más tarde. Incorpora en esta preocupación la formación de un maestro capaz de cumplir con su función política, entendiendo lo político como la acción encaminada a solucionar los problemas que enfrenta en su ejercicio profesional y como agente socio cultural en su comunidad haciendo del maestro el leader social que reclama nuestra sociedad. Llevar a la práctica la escuela social fue para Encinas apostar por un modelo de Escuela Rural. (Marrou, 2012, p.8)

Encinas exigía:

Superar el doloroso cuadro que presenta esa juventud triste y apática llevando una vida homogénea sin que las incertidumbres del porvenir aguijone en su alma. (Morrou, Encinas Franco, José Atonio, 2012, p. 8)

Entre otros temas consideraba que el maestro debía ser no sólo un enseñador sino un líder de desarrollo y promoción social. La escuela no debe desarraigar al indígena de su vida social y de su concepción del mundo. 
Al respecto, Gamaliel Churata (Arturo Peralta), prologando un ensayo de Encinas, titulado "Escuela Nueva en el Perú", escribe:

Este libro es acaso el mensaje de un hombre reclamado por las necesidades del país. A través de la exposición de su doctrina pedagógica - exposición llena de vivacidad, de energía, colorido y pasión - se descubre al estadista, al hombre signo en quien deben mirar los hombres nuevos la posible realidad de un conductor que en la sabiduría empírica, hace radicar la mayor riqueza de una cultura. (Morrou, 2012, p. 11)

Encinas, defendió la escuela laica y libre; aprender haciendo, sin que tuvieran al profesor como un supervisor con látigo. La lectura debería ser sobre temas interesante de su ambiente "como morada", con palabras normales, sentido crítico de la historia, no tanto repitiendo personajes y fechas. Los estudiantes debían conocer su morada, salir del local de la escuela al campo. Debían seguir el método experimental, esto es: observar, comparar, explicar, generalizar. El maestro no debía hacer sino completar lo que el alumno había captado en su contacto con la realidad. El sistema debía tener una orientación pragmatista.

La evaluación debía realizarse a partir de ejecución de proyectos. Luego enseñando a abstraer y generalizar. Encinas, sostuvo que la educación oficial, no hacía sino concebir al indígena como un "paria". Así mismo, la escuela no debía ser un lugar hostil para el adulto. El educando debía aprender de su vida real, de la participación en todas las actividades de la comunidad. La escuela debe ser una aldea de convivencia, de producción, de fiesta.

\section{LA ORIENTACIÓN DE LA FILOSOFÍA DE RODOLFO KUSCH}

En la lectura de las obras del autor se pueden encontrar la siguiente secuencia:

a. En primer lugar Kusch siente y piensa como latinoamericano e inicia su exposición haciendo observación crítica de la realidad y de los temas tratados; para ello entra en contacto con el hombre real, 
con quien dialoga frente a frente, comunicándose directamente o por medio de un intérprete, como cuando visitó Qollana ayllu, en Oruro, Bolivia; Así mismo, Kusch se informa también, recurriendo a textos escritos sobre nuestras raíces culturales y pensamiento propio además de la información de su momento.

b. La información recibida a manera fenomenológica es interpretada recurriendo a su formación existencialista y muy personal, sobre todo, cuando interpreta el existir (estar siendo no mas) del hombre andino, principalmente al qolla o aimara y, luego del runa (hombre inka).

c. Plantea soluciones a partir de la información recogida de primera mano y de la información bibliográfica, y de la interpretación recogida de sus informantes académicos.

d. A partir de la experiencia de campo y de la información directa e indirecta, construye, una concepción del mundo andino qolla e inka. Sin embargo sus conclusiones no los realiza a manera de dogma sino, abiertos para continuar con las investigaciones. Por ello, no sólo los argentinos, peruanos, bolivianos estamos involucrados en ello sino todos los latinoamericanos; parafraseando a Juan Cepeda podemos decir que "la filosofía tiene siempre una mirada universal", pero, siempre desde aquí y desde nosotros. Kusch, nos interpela a todos, principalmente a todos aquellos que tenemos raíces indígenas, no sólo de América Latina, porque no sólo existen indígenas en esta parte del mundo.

El pensamiento de Kusch, lleva inserta un mensaje educativo general, que incluye a los niños a los jóvenes y a los adultos; toda vez que los adultos también, necesitamos "nacer de nuevo" para ubicarnos en la pacha americana, esto es, en el espacio, tiempo, naturaleza y mundo, americanos. Necesitamos partir de lo positivo de nuestras raíces culturales, de las prácticas que hicieron posible una vida propia sustentada en el trabajo como una actividad religiosa festiva de realización y de esperanza.

En primer lugar pensamos que, somos los adultos quienes debemos cambiar, "nacer de nuevo" (andragogía), para cambiar la visión de nuestros jóvenes y niños. Luego, en las universidades, una nueva efebología, para para los adolescentes varón y una Hebelogia para la formación de las adolescentes damas. (Vease: http://www.significadode.org/efebologia.htm)

Los contenidos de esta nueva orientación deben ser de carácter intercultural, de información histórica, social, científica y cultural, de carác- 
ter andino, latinoamericano y universal; dichos temas deben estar contenidos de manera transversal. Debemos insertarnos en la "línea del tiempo", en la historia universal, pero primero zambulliéndonos en nuestra cultura original, básica; cimentando nuestra cultura en un piso firme, para investigar e informar el conocimiento vivo de nuestra realidad y, también recibir los conocimientos que vienen de otras pachas.

Rodolfo Kusch, hace un cuestionamiento profundo de lo que debe ser el pensamiento y la filosofía latinoamericana y con ella la indígena. Señala más profunda y auténticamente que José Carlos Mariátegui y la pedagogía del oprimido.

Pedro Zulén y Antonio Encinas, en el Perú, plantearon una educación del indígena a partir de una filosofía de la educación nativista, empleando para ello, el idioma propio y enseñándole primero a satisfacer sus necesidades básicas, mediante la actividad productiva, en su propio medio ambiente. Los indígenas deben primero conocer y administrar su medio ambiente, luego proyectarse a la región y por último involucrarse en la vida y proyectos de la nación y el Estado.

Rodolfo Kusch, va más allá, no sólo cuestiona la educación indígena sino, la cultura y la filosofía "oficial" de toda Latinoamérica, por ser éstas imitativas y repetitivas; señala a quienes pretenden demostrar aires de superioridad intelectual, repitiendo teorías y apreciaciones del mundo ajeno e ignorando el nuestro.

Con ello, no queremos decir que las concepciones de los intelectuales occidentalizados no fueron o no son significativos, de ninguna manera; pero debemos denunciar que no fueron efectivos para el mundo latinoamericano. La cultura y el pensamiento latinoamericano también tiene sus detalles sutiles muy propios, en lo que respecta a su pensamiento, en su español latinoamericano, en que quechua-ñol, aimara-ñol, guaraní-ñol y en el lenguaje autóctono de cada una de las naciones que tienen idiomas y lógicas propias; espacio propios, así como historia y dioses propios. 


\section{LA PROPUESTA DE EDUCACIÓN INDIGENISTA DE RODOLFO KUSCH}

En el II tomo de sus obras, bajo el título de completas Kusch propone:

La búsqueda de un pensamiento indígena no se debe sólo al deseo de exhumarlo científicamente, sino a la necesidad de rescatar un estilo de pensar que, según creo, se da en el fondo de américa y que mantiene cierta vigencia en las poblaciones criollas. (Kusch, 2007, p. 259)

El rescate de un pensar indígena es importante porque abre la comprensión de esta América poblada últimamente por ideologías dispares. Supuestamente científicas, como es el de los sociólogos, o economista, como el de ciertas ramas demasiado limitadas del marxismo o presuponiendo ideales democráticos donde no corresponde o sino determinando simplemente formas religiosas; todo ello carece de sentido, porque nunca dice realmente la verdad. Son estos canales por donde el americano suele escurrir sus opiniones, sin darse cuenta que la orientación que da a su pensamiento constituye el principal impedimento para comprender el estilo de vida real al cual pertenece. (Ibídem, p. 259-260)

Kusch, manifiesta que el mundo occidental gira sobre otro eje que la del campesino; así aclara, en su obra: “...En el presente trabajo no quise tomar en cuenta tampoco (Ibídem, $p$. 261) los últimos aportes de la antropología y la psicología...”

...entretenerse con el estructuralismo o con la psicología profunda, o con su epígonos como Mircea Eliade y tantos más, es, en estos momento, en Argentina, uno de los entretenimientos más eficaces, quizá por el hecho de creer que se está jugando con la última explicación del mundo. (Ibídem p. 261)

Considero que este pensamiento nos abre la comprensión de los problemas americanos $\mathrm{y}$, por consiguiente, la segunda parte del libro está dedicada a medir las posibilidades que dicho pensamiento ofrece. A su vez, no podía estar ausente en este trabajo el esbozo de un pensamiento americano que gire en torno al concepto estar. Creo que dicho término logra 
concretar el verdadero estilo de vida de nuestra América, en la cual entrarían blancos y pardos, y ofrece desde un punto de vista fenomenológico, una inusitada riqueza. Va implícito en dicho término esa peculiaridad americana a partir de la cual recién habremos de ganar, si cabe, nuestro verdadero lugar, y no esa penosa universalidad que todos pretendemos esgrimir inútilmente. (Ibídem, p. 262).

Señalada la propuesta indigenista de Rodolfo Kusch, seguidamente pasaremos a exponer la introducción a la propuesta kuschiana de Oscar Hernández.

\section{INTRODUCCIÓN A LA PROPUESTA PEDAGÓGICA DE ÓSCAR HERNÁNDEZ}

En la Introducción de su artículo: "Reflexiones sobre la educación latinoamericana desde los planteamiento de Rodolfo Kusch" bajo el subtítulo de Un modelo pedagógico situacional, hermenéutico y transformado para Latinoamérica, muy acertadamente propone: (Hernández, 2015, p. 193 ss.)

a. Ubicar al hombre en su propia realidad.

b. Interpretar su realidad desde el aquí, y desde su propio mundo tome una posición hermenéutica y lo valore para transformarlo;

c. ...el hombre debe construir su propia realidad y es partir de allí que se convierte en un ser educable: "homo educandus" (Hernández, p. 194)

d. "homo educandus", que posee la capacidad de aprender, de saber y de conocer, «pero, ¿qué es lo que tenemos que saber?, he aquí nuestro problema,...

e. Sobre la manera de enseñanza aprendizaje, (mediante la multimedia actual),... Hernández opina que no es sino, la recepción de un cumulo de ideas y conceptos en cantidad, donde el análisis y profundidad de la realidad se vuelven superfluos; la multimedia nos ofrece un océano de conocimientos pero con un centímetro de profundidad.

f. Propone que, antes que ser marxistas o existencialistas, por decir algo, debemos ser nosotros mismos y pensar como somos. (Ibídem, p. 201) 
g. Como Alberto Buela, sostiene que el objetivo de la filosofía de Kusch es "pensar lo propio de América" y que la posibilidad de un pensamiento propio está encarnada en el pensamiento popular. Así el pueblo es "el lugar filosófico desde donde se realiza y se resuelve la genuina pregunta filosófica" (Buela, On line). (Ibídem, p. 197)

h. Para Kusch «...preguntar por un pensamiento popular, encubre la posibilidad de descubrir un pensamiento propio." (Ibídem, p, 197)

Hernández se pregunta: ¿Al buscar un pensamiento propio una filosofía propia no estaríamos entrando a un sectarismo como Cuba y Venezuela?

Óscar Hernández sostiene que frente a estas interrogantes se abre la necesidad de una Pedagogía Latinoamericana, que permita guardar el equilibrio entre nuestras tradiciones culturales y los cambios actuales tanto de la sociedad, la política y de la educación misma.

Con la introducción arriba planteada Hernández plantea algunas características a pedagogía, como sigue: "Por tal motivo quisiera plantear algunas características de ésta pedagogía Situacional, Hermenéutica y Transformadora para Latinoamérica” (Ibídem, 198 ss)

\section{CARACTERÍSTICA DE LA PEDAgOgíA PROPUESTA POR ÓSCAR HERNÁNDEZ}

Hernández señala que las características de una pedagogía en el sentido kuschiano deben ser:

a. Una pedagogía, ecuánime: Esto es, evitar llegar a extremos en los planteamientos; reconocer la importancia de los aportes dados desde occidente y desde otras culturas, pero no colocarlos por encima de la producción intelectual y cultural de nuestra América latina.

b. Abierta: En América Latina, no solo se debe hacer filosofía desde lo político y lo social, sino también cabe hacer una filosofía desde la misma naturaleza, desde el hombre latinoamericano, desde sus creencias religiosas, y por qué no desde el Ser.

c. Hermenéutica: Está llamada a hacer una interpretación correcta de la realidad latinoamericana, pero a la vez fenomenológica, es decir ver las cosas tal y como se presentan en su propia realidad. Esta 
capacidad interpretativa del ser humano, exige que al hacerse desde nuestro contexto, una adecuada visión de sí mismo, de las cosas y de las posibilidades del hombre latinoamericano.

d. Situacional. Reconocer la ubicación del hombre latinoamericano en un contexto tanto inmediato como extenso. Inmediato, en cuanto que ve al hombre desde su propia tierra, génesis y su arraigo cultural. Extenso, en cuanto ubica al hombre como un ciudadano del universo, lo cual no se le puede negarse la posibilidad de Ser en el Mundo sin perder su propia originalidad,

e. Propositiva: Iluminar los sistemas educativos en cada uno de los países latinoamericanos, incentivando la posibilidad de realizar un proyecto latinoamericano de Pedagogía autóctono, con fundamentos teóricos y conceptuales surgidos del pensamiento de autores de nuestras naciones.

f. Filosófica: Siempre reflexiva, en continua búsqueda de soluciones a los interrogantes y situaciones que se van presentando en la construcción de una pedagogía situacional hermenéutica y transformadora. La filosofía no puede seguir viéndose como una simple actitud o visión diferente frente a la vida, es también una posición que debe ir de la teoría a la práctica, de la reflexión a la acción, del pensamiento a la realidad. No puede quedarse en simples discursos estériles sobre la realidad, debe iluminar el adecuado modo de actuar en la sociedad frente a las diversas circunstancias que se enfrenta el hombre latinoamericano.

g. Humanista: Que valore al ser humano por lo que es en su esencia, en su libertad y sus posibilidades de realización, desde su propio estar y ser en este mundo. A la vez que lo reconozca de manera integral en su pluridimensionalidad física, espiritual, familiar, sexual, comunitaria, política, religiosa y social.

h. Actual: Hacer buen uso de la tecnología y la informática, reconociendo en ella un medio y no un fin, estar atenta y vigilante de las innovaciones de la ciencia, pero asumirlas con criterio para sopesar el impacto en el hombre.

En tal sentido Oscar Hernández concluye su propuesta indicando que:

Sólo en la medida que se reconozca estos ocho puntos, y quizás algunos más, podemos hablar de una pedagogía que transforma la vida del hombreen su pensar, su sentir y su actuar, le construye como persona y le edifica en comunidad como ser social que aprende y se educa con los demás y para 
los demás, que persigue fines en común a través de medios propios que enriquecen a los otros, en la medida que recibe de éstos. (Ibídem, p. 202)

Nosotros pensamos que ocho puntos, son difíciles de atacar y acatar, quizá al inicio deberíamos reducir a unos cuatro, con miras a su aplicación efectiva. Por los años sesenta del siglo pasado, los físicos estuvieron afanados en la búsqueda de la Ley General de Unificación (GUT), al principio alguien propuso que hechos podían explicarse con unos veinte principios; la propuesta no fue aceptada por que eran muchos, entonces acordaron que deberían reducir la explicación a pocos principios; pensaron que lo mejor sería: "poder explicar la totalidad de los hechos conocidos y por conocer mediante una sola teoría". (Miró-Quesada, 1989, p. 67). Efectivamente, los físicos aceptaron que los fenómenos del macrocosmos y de la mecánica cuántica (microcosmos) podían explicarse mediante seis principios. Por entonces, consideraron que se había logrado un gran avance en la búsqueda de la Ley General de la Unificación.

Basados en la concepción indígena del mundo pensamos que podría reducirse la propuesta de los principios pedagógicos, como sigue a continuación:

1 La Teoría de la Unificación. GUT permitió unir en una fórmula matemática la fuerza electro débil con la fuerte, se había logrado un avance espectacular hacia la unidad, la meta suprema del conocimiento racional. (p. 68), “...pero luego ya no se trataba de unificar dos campos sino cuatro. Fue necesario considerar, por eso, un conjunto mayor de dimensiones. Los primeros resultados fueron tan pasmosos como descorazonadores: la teoría funcionaba de manera consistente en un espacio-tiempo de veintiséis dimensiones. Esto era demasiado... veintiséis dimensiones eran una monstruosidad. Por eso la teoría fu rechazad por la comunidad científica... poco tiempo después de haberse demostrado que la teoría funcionaba con veintiséis dimensiones, se establecido que también funcionaban con diez,...pero 10 era un número aceptable...Uno de los aspectos menos logrado es la explicación de la existencia de diez dimensiones pues sólo pueden percibirse y medirse las cuatro dimensiones conocidas. Hasta el momento nadie ha podido explicar por qué hay seis más...La explicación más aceptada es la planteada en la hipótesis de Klein: las seis dimensiones restantes se han enrollado sobre si mimas y se han vuelto tan pequeñas que no pueden detectarse mediante ningún aparato. Se cree que en los comienzos del universo, unas millonésimas de segundos después de la gran explosión, todas la dimensiones estaban enrolladas y sólo se desplegaron las cuatro conocidas" (Miró Quesada, 1992, pp. 84-86.) 


\section{NUESTRA PROPUESTA A MANERA DE CONCLUSIÓN:}

Como habíamos indicado, consideramos que la propuesta de Oscar Hernández es plausible; pero consideramos que son muchas, como para que estas sean tomadas como principios orientadores de la educación indígena. Lo que nosotros vamos a hacer de su propuesta es indigenizarlo como sigue:

a. La educación debe ubicarnos en su propia pacha, para que seamos "nosotros mismos". Nuestro currículo educativo debe comenzar con el conocimiento de nuestra pacha y el empleo de la técnica propia y los aportados por la tecnología de última generación, con el objetivo de llegar a un "sumaq kawsay" (vida plena). José María Arguedas sostenía que nosotros no podremos salir adelante sin la ciencia y la tecnología extranjeras. Los gringos nos ganan en ciencia y tecnología, pero no en arte.

b. Debemos hacer que el indígena aprecie y valore su cultura, además de empoderarlo con las técnicas que él conoce y, con las técnicas actuales, las mismas que deben ser enseñadas con el ejemplo.

c. El indígena tiene como tácita obligación aprender, a vivir como lo hizo y lo hace su familia nuclear y su familia extendida, no hay alternativa. Quien no ha aprendido los usos y costumbres y las formas de trabajo de la comunidad, no tiene cabida en ella. Aquí también están, las obligaciones de los padres y de la propia comunidad, enseñar a las nuevas generaciones, la cultura material y espiritual de la comunidad. Como sostuvimos en el punto anterior, el aprendizaje y la enseñanza en la comunidad no tiene alternativa. Por naturaleza el indígena es un "homo educandus" como bien afirma Óscar Hernández. En el idioma inka, yachay es el verbo que al mismo tiempo significa aprender y saber. Yachay y yachachiy son las distintas caras de la misma moneda. La nueva generación, tiene que saber: cómo vivir, cómo trabajar, cómo relacionarse con los demás y con los dioses. Además, actualmente, todo indígena debería saber leer y escribir, primero en su propio idioma y luego en el idioma oficial, importado.

d. Los filósofos latinoamericanos, como también sostuviera David Sobrevilla, debemos tener conocimiento de la filosofía europea y americana, pero también hacer, lo nuestro". Hacer filosofía de nuestra pacha. Gamaliel Churata, pensaba como Kusch, y sostenía que si el logos griego es la fuente y artífice de la filosofía occidental, el logos aimara o quechua deben ser de la filosofía y la cultura andina propia. 
Por último, en todo nivel debemos exigir al Estado, primero que tome consciencia de que la educación es una inversión a largo plazo; segundo a que esta inversión no sea ejecutada sólo para la educación en el idioma oficial, sino y sobre todo, en la formación de las naciones indígenas, contrariamente, los ecos de estas reuniones y congresos no trascenderán los muros de nuestros recintos académicos.

\section{REFERENCIAS BIBLIOGRAFÍCAS}

Hernández, Ó. (2015). En: Estar y Ser. Aproximaciones hermanéuticas y ontológicas a la obra de Rodolfo Kusch. Saarbrucken, Alemania: Editorial Académica Española.

Kusch, R. (2007). Obras Completas. Tomo II. Santa Fe, Argentina: Editorial Fundación Ross.

Mariátegui, J. C. (1980). 7 Ensayos de Interpretación de la Realidad Peruana. Lima, Perú: Amauta.

Miró Quesada, F. (1992). Las Supercuerdas. Lima, Perú: Ediciones de "El Comercio".

Morrou, A. (2012). Encinas Franco, José Antonio. Chosica, Lima, Perú: Editorial Universitaria de la Universidad Nacional de Educación Enrique Guzmán y Valle. 
